\title{
Identificação das mudanças na mastigação e deglutição de indivíduos submetidos à glossectoma parcial
}

\section{Identification of chewing and swallowing changes in individuals submitted}

\author{
to partial glossectomy
}

\author{
Laura Cristina Sales de Oliveira ${ }^{1}$, Camila Alves Vieira ${ }^{2}$, Marta Helena Marques Mota ${ }^{3}$, Patrícia Vieira Salles ${ }^{4}$, José \\ Maria Porcaro Salles 5 , Camila Queiroz de Moraes Silveira Di Ninno ${ }^{6}$, Ana Teresa Brandão de Oliveira e Britto
}

\begin{abstract}
RESUMO
Objetivo: Identificar as alterações de mastigação e deglutição decorrentes da cirurgia curativa do câncer de língua, com extensão inferior a 50\% da dimensão da língua e sem comprometimento do soalho da boca e da base da língua. Métodos: Foram realizadas avaliações das funções de mastigação e deglutição em nove pacientes, seis homens e três mulheres, no período pré-operatório, aplicando-se um protocolo específico. No pós-operatório mediato, três semanas após a cirurgia, cinco pacientes foram reavaliados, quatro homens e uma mulher, seguindo o mesmo protocolo. Para verificar a significância dos resultados foi utilizado o teste não paramétrico de Kruskall Wallis (Teste H). Resultados: Comparando-se os achados do pré-operatório com os achados do pós-operatório encontramos, de forma significativa $(\mathrm{p}<0,05)$, mudança da via de alimentação, que passou de uma alimentação exclusivamente oral, para uma alimentação exclusivamente enteral. Também houve mudança significativa na eficiência mastigatória, que passou a ser ineficiente em todos os pacientes. Percebeu-se, de forma significativa, a dificuldade dos pacientes em manipular o bolo alimentar durante o processo de mastigação, gerando dificuldade na formação de um bolo coeso. Isso demonstra que a cirurgia influencia na realização desta função, ou seja, a perda de parte da língua compromete o processo de mastigação. A deglutição também foi prejudicada pela cirurgia, uma vez que o teste demonstrou de forma significativa a presença de estase oral, após a deglutição e movimentos compensatórios de cabeça para a deglutição de alimentos sólidos. Conclusão: Os pacientes submetidos à glossectomia parcial apresentam mudanças na mastigação e deglutição decorrentes do tratamento cirúrgico.
\end{abstract}

Descritores: Neoplasias da língua/complicações; Neoplasias bucais/complicações; Glossectomia; Sistema estomatognático; Transtornos de deglutição/etiologia; Mastigação; Fonoterapia

Trabalho realizado no Curso de Especialização em Motricidade Orofacial da Pontifícia Universidade Católica de Minas Gerais - PUC Minas - Belo Horizonte (MG), Brasil.

(1) Especialização em Motricidade Orofacial pela Pontifícia Universidade Católica de Minas Gerais - PUC Minas - Belo Horizonte (MG), Brasil; Fonoaudióloga do Hospital Alberto Cavalcanti, Fundação Hospitalar do Estado de Minas Gerais FHEMIG - Belo Horizonte (MG), Brasil.

(2) Especialização em Motricidade Orofacial pela Pontifícia Universidade Católica de Minas Gerais - PUC Minas - Belo Horizonte (MG), Brasil; Fonoaudióloga do Centro Médico Integrado Santo Ivo - Belo Horizonte (MG), Brasil.

(3) Especialização em Motricidade Orofacial pela Pontifícia Universidade Católica de Minas Gerais - PUC Minas - Belo Horizonte (MG), Brasil; Fonoaudióloga das Prefeituras Municipais de Bonfim e Rio Manso (MG), Brasil.

(4) Mestre, Professora Assistente do Curso de Fonoaudiologia da Pontifícia Universidade Católica de Minas Gerais - PUC Minas - Belo Horizonte (MG), Brasil; Membro do Grupo de Cirurgia de Cabeça e Pescoço do Instituto Alfa de Gastroenterologia do Hospital das Clínicas da Universidade Federal de Minas Gerais - UFMG - Belo Horizonte (MG), Brasil.

(5) Mestre, Professor Adjunto do Departamento de Cirurgia da Faculdade de Medicina da Universidade Federal de Minas Gerais - UFMG - Belo Horizonte (MG), Brasil.

(6) Doutora, Professora Assistente do Curso de Fonoaudiologia da Pontifícia Universidade Católica de Minas Gerais - PUC Minas - Belo Horizonte (MG), Brasil.

(7) Pós-graduanda em Lingüística pela Pontifícia Universidade Católica de Minas Gerais - PUC Minas - Belo Horizonte (MG), Brasil; Professora do Curso de Fonoaudiologia e de Especialização em Motricidade Orofacial da Pontifícia Universidade Católica de Minas Gerais - PUC Minas - Belo Horizonte (MG), Brasil.

Endereço para correspondência: Laura Cristina Sales de Oliveira. R. Aurélia, 111, Boa Vista, Belo Horizonte - MG, CEP 31070-480. E-mail: lauracso@hotmail.com

Recebido em: 7/1/2008; Aceito em: 8/8/2008

\section{INTRODUÇÃO}

O sistema estomatognático é composto por dois grupos distintos de estruturas orais, a saber: as estruturas estáticas ou passivas e as estruturas dinâmicas ou ativas. O primeiro grupo é representado pelos arcos osteodentários, maxila, mandíbula - relacionados entre si pela articulação temporomandibular -, outros ossos cranianos e o osso hióide, e o segundo grupo pela unidade neuromuscular, que mobiliza as partes estáticas do primeiro grupo ${ }^{(1)}$. Estas estruturas, quando equilibradas e controladas pelo sistema nervoso central, são responsáveis pelo funcionamento harmônico da face e pelas funções estomatognáticas de respiração, mastigação, sucção, deglutição e fala ${ }^{(1)}$.

A mastigação é um ato fisiológico e complexo, que envolve atividades neuromusculares e digestivas sequienciais, e que depende dos padrões de crescimento, desenvolvimento e amadurecimento do complexo crânio-facial, do sistema nervoso central e das guias oclusais, o que é fundamental para que as funções de deglutição e fala, sejam desempenhadas com eficiência e precisão, e para que não ocorram distúrbios miofuncionais ${ }^{(1)}$. Pode ser classificada em bilateral alternada ou unilateral e dividida em três fases: incisão ou mordida, tri- 
turação e pulverização - processo responsável pela formação de um bolo alimentar homogêneo e coeso, juntamente com a eficiência dos golpes mastigatórios ${ }^{(2)}$.

A deglutição é uma seqüência reflexa de contrações musculares ordenadas, que leva o bolo alimentar ou líquidos da cavidade oral até o estômago, sendo, portanto uma atividade neuromuscular complexa e integrada ${ }^{(1)}$. Assim como a mastigação, a deglutição também pode ser dividida em fases: antecipatória, preparatória oral, oral propriamente dita, orofaríngea e esofágica ${ }^{(3)}$. Alguns autores, no entanto, não consideram a fase antecipatória, e acreditam que a deglutição é composta por apenas quatro fases, sendo que qualquer alteração neste processo neuromotor, responsável pelo transporte do alimento da boca até o estômago, é classificada como disfagia ${ }^{(4)}$.

Existem fatores com as neoplasias de cavidade oral, que podem comprometer o processo normal de mastigação e deglutição. A mastigação e a deglutição são funções da cavidade oral, que dão suporte à vida e que estão intimamente relacionadas à nutrição. Embora a perda da mastigação possa ser compensada pela mudança da consistência alimentar apropriada, a perda da deglutição é uma condição ameaçadora à vida ${ }^{(5)}$.

Os dados do Instituto Nacional do Câncer (INCA) do ano de 2005 mostraram que o câncer de boca foi o oitavo tipo de tumor maligno mais comum no Brasil, tendo sido registrados 9.985 casos novos em homens e 3.895 em mulheres. A estimativa de incidência para o ano de 2006 foi de 10.060 casos novos em homens e 3.410 em mulheres. Estima-se que na cidade de Belo Horizonte ocorrerão 140 casos novos por ano, para cada 100.000 habitantes $^{(6)}$.

O câncer de boca está relacionado principalmente ao tabagismo e etilismo ${ }^{(7)}$, mas fatores como idade, sexo, raça, hereditariedade e irritabilidade crônica, também podem ser considerados como fatores predisponentes ao seu desenvolvimento $^{(8)}$.

O tratamento preconizado para o câncer de boca envolve diferentes modalidades terapêuticas, tais como: a cirurgia, a radioterapia e a quimioterapia, e depende também da localização, do grau de malignidade e do estadiamento do tumor, assim como, das condições físicas do paciente ${ }^{(9-10)}$. Em princípio o tratamento de escolha para as neoplasias avançadas inclui sempre procedimentos cirúrgicos associados à radioterapia ${ }^{(10-11)}$.

A despeito dos avanços das técnicas de diagnóstico e tratamento, a sobrevida dos pacientes portadores de neoplasias de cabeça e pescoço tem aumentado apenas marginalmente.

Por ser uma doença que resulta em alta morbidade e mortalidade, faz-se necessário o diagnóstico precoce e imediato encaminhamento ao tratamento, pois, quando diagnosticada em sua fase inicial, o tratamento cirúrgico será suficiente para curar a maioria dos pacientes ${ }^{(12-13)}$. Quanto mais precoce for o diagnóstico, menor será a extensão cirúrgica, gerando, portanto, menor comprometimento funcional. Porém, apesar de ser um tumor de fácil diagnóstico devido à acessibilidade da cavidade oral e à presença de lesões precursoras bem definidas, o câncer de boca é geralmente negligenciado pelos profissionais da saúde ${ }^{(14-15)}$.

Quando uma doença como o câncer se manifesta na boca, é fundamental realizar o acompanhamento fonoaudiológico pré e pós-tratamento. $\mathrm{O}$ acompanhamento visa orientar e tra- tar o paciente, tendo em vista as seqüelas ocasionadas pelas modalidades de tratamento, uma vez que estas trarão prejuízos para o bom funcionamento das funções estomatognáticas, principalmente a mastigação e deglutição, e conseqüentemente, o processo de alimentação, que deve ser priorizado na atuação fonoaudiológica, a fim de manter uma melhor qualidade de vida para o indivíduo ${ }^{(16)}$.

É importante que o fonoaudiólogo saiba exatamente qual a modalidade terapêutica preconizada para que possa fazer uma avaliação adequada e traçar o plano terapêutico específico ${ }^{(17)}$. Devemos ressaltar que, nos casos de cirurgias de cavidade oral, duas cirurgias classificadas com o mesmo nome podem deixar alterações fonoaudiológicas diferentes, de acordo com o local e a extensão da lesão. Cirurgias parciais de ponta de língua deixam seqüelas diferentes daquelas realizadas em borda lateral, assim como, cirurgias nas quais são retiradas porções menores que $50 \%$ da língua deixam seqüelas funcionais menores quando comparadas às cirurgias nas quais são retiradas porções maiores que $50 \%$, como nas glossectomias quase totais $^{(17)}$.

As principais seqüelas do tratamento cirúrgico relacionadas ao processo de mastigação e deglutição são as dificuldades na formação, propulsão ântero-posterior e retenção do bolo alimentar, aumento do tempo de trânsito oral, redução do movimento vertical e lateral da mandíbula, estase de alimentos nos sulcos: anterior e lateral, palato duro (alimentos mais espessos) e valécula, e aumento do número de deglutições ${ }^{(18-19)}$.

Os exercícios de mobilidade de língua durante a radioterapia, em pacientes submetidos à cirurgia, podem reduzir a formação de tecidos fibróticos na cavidade oral e promover melhor limpeza faríngea ${ }^{(20)}$.

A partir do que foi exposto anteriormente é possível perceber a importância do acompanhamento fonoaudiológico em todo o processo do tratamento do câncer de língua.

O objetivo desta pesquisa foi identificar as alterações na mastigação e deglutição mais comuns, decorrentes de cirurgia curativa do câncer de língua, avaliando pacientes no pré e pós-operatório.

Esta pesquisa foi aprovada pelo Comitê de Ética em Pesquisa (CEP) da Pontifícia Universidade Católica de Minas Gerais (CAAE 0037.0.213.000-05), e os pacientes, antes de serem avaliados, foram esclarecidos sobre os objetivos deste estudo e assinaram o Termo de Consentimento Livre e Esclarecido.

\section{MÉTODOS}

Participaram desta pesquisa indivíduos com câncer primário de língua, seis homens e três mulheres, todos com indicação de realização de glossectomia parcial, sem ressecção total de assoalho de boca e base de língua, com idade variando de 40 a 72 anos (média de 58 anos). Todos os participantes deste estudo foram submetidos à cirurgia curativa do câncer de língua, com ressecções parciais, nas quais foram retidas porções menores que $50 \%$ desta estrutura. As lesões estavam localizadas em borda lateral de língua, direita ou esquerda e tiveram a reconstrução realizada com fechamento primário ou com retalho miocutâneo de peitoral maior. Os informantes foram encaminhados aos pesquisadores de consultórios particulares, 
localizados na cidade de Belo Horizonte.

Os pacientes com tumores que envolviam apenas parte da língua foram o foco desta pesquisa, devido à importância da língua nas funções de mastigação e deglutição. Por esse motivo, foram excluídos pacientes que seriam submetidos a ressecções muito extensas, como glossectomia quase total, glossectomia total ou laringectomia total.

Os pacientes foram submetidos a uma anamnese específica para a identificação e conhecimento da história da moléstia. Em seguida, foram submetidos à avaliação fonoaudiológica para verificar força, mobilidade e sensibilidade dos órgãos fonoarticulatórios e o desempenho das funções de mastigação, deglutição e fala.

Estes procedimentos foram aplicados em dois momentos distintos, no pré-operatório e no pós-operatório. A avaliação pós-operatória foi realizada três semanas após a realização da cirurgia, quando os pacientes já não apresentavam edema intra-oral, apenas um discreto edema de face, conseqüência do esvaziamento cervical, e ainda, não estavam em tratamento radioterápico, pois a radioterapia poderia influenciar os achados funcionais ${ }^{(16)}$.

Foram avaliados nove pacientes no pré-operatório e, destes, cinco foram reavaliados no pós-operatório. Os quatro pacientes que não foram reavaliados no pós-operatório tiveram a cirurgia programa alterada, no per-operatório. Durante o ato cirúrgico estes pacientes necessitaram de ressecções maiores, em função da invasão do tumor, o que era um fator de exclusão. Desta forma, iniciamos nosso estudo com uma amostra constituída por seis homens e três mulheres e o finalizamos com quatro homens e uma mulher.

A escolha destes momentos distintos - pré-operatório e pós-operatório - justifica-se pela necessidade de avaliação das funções de mastigação e deglutição com a presença da lesão (pré-operatório), para que padrões inerentes ao sujeito fossem identificados e pudessem ser comparados com os padrões do mesmo sujeito no pós-operatório, permitindo que somente a variável cirurgia fosse analisada.

Os pacientes foram fotografados e filmados por meio de uma câmera digital da marca Sony®, modelo PSC-P73, após autorização prévia, com a finalidade de facilitar a análise dos dados.

$\mathrm{Na}$ avaliação, procurou-se identificar possíveis alterações dos músculos da mastigação, qual a via de alimentação utilizada, assim como, descrever a mastigação e a deglutição apresentadas pelos informantes antes e depois da cirurgia. Considerou-se como normal o padrão de mastigação descrito por Bianchini ${ }^{(2)}$ e o de deglutição descrito por Macedo Filho ${ }^{(4)}$. Os materiais utilizados para avaliação das funções foram água (líquido), Danette (pastoso grosso), Bliss (pastoso fino) e Bisnaguinha Seven Boys (sólido).

Para a realização desta pesquisa, consideramos como mastigação eficiente, aquela que permitia a formação de um bolo alimentar homogêneo e coeso, e como mastigação ineficiente, aquela que deixava o bolo alimentar com resíduos, ou seja, no bolo deglutido havia pedaços de alimento não triturado e pulverizado. Em relação à deglutição, consideramos como deglutição alterada a presença de movimentos compensatórios de cabeça ou contração da musculatura perioral, presença de resíduos alimentares em boca após a deglutição, assim como, presença de aspiração do alimento ingerido, independente da consistência.

A análise estatística dos resultados das avaliações foi realizada por meio da aplicação do teste não paramétrico de Kruskall Wallis (Teste H), com nível de significância de 5\%. Os dados das anamneses e dos achados clínicos foram analisados de forma descritiva.

\section{RESULTADOS}

As informações coletadas na anamnese evidenciaram que $67 \%$ dos participantes da pesquisa eram do sexo masculino e $33 \%$ do sexo feminino, com média da idade de 58 anos, idade esta variando entre 40 e 72 anos. Todos os pacientes tiveram como diagnóstico histológico o carcinoma espinocelular e $67 \%$ apresentaram metástase em linfonodos cervicais.

Do total da amostra, apenas $22 \%$ dos pacientes relataram apresentar alterações intra-orais como aftas, e distúrbios do aparelho digestivo, como gastrite. Com relação aos hábitos viciosos, $89 \%$ da amostra faziam uso de tabaco associado ao álcool, 55\% faziam uso constante de alimentos condimentados e $77 \%$ tinham preferência por alimentos bem quentes. Dos pacientes entrevistados, $89 \%$ relataram odinofagia durante a deglutição em função da lesão, enquanto $56 \%$ referiram dificuldades na mastigação.

Os dados obtidos na avaliação fonoaudiológica pré-operatória demonstravam que todos os pacientes alimentavam-se exclusivamente por via oral. Com relação à mastigação, cinco pacientes $(56 \%)$ apresentaram mastigação eficiente, enquanto quatro pacientes (44\%) apresentaram mastigação ineficiente e apenas um paciente $(11 \%)$ apresentou diminuição da mobilidade de língua. Com relação à deglutição, a avaliação préoperatória mostrou que dois pacientes $(22 \%)$ apresentaram movimentos compensatórios de cabeça durante a deglutição de sólidos e nenhum apresentou sinais clínicos de aspiração.

Já a avaliação na terceira semana pós-operatória evidenciou que $100 \%$ dos pacientes, após a realização da glossectomia parcial, alimentavam-se por sonda nasoentérica e apresentaram mastigação ineficiente. Com relação à manipulação do bolo alimentar dentro da cavidade oral, dois pacientes (40\%) não apresentaram dificuldade de manipulação, enquanto três $(60 \%)$ a apresentaram, apesar da redução de mobilidade de língua em quatro pacientes $(80 \%)$. Em relação à deglutição, dois pacientes $(40 \%)$ apresentaram estase oral de alimentos após a deglutição e quatro $(80 \%)$ apresentaram movimentos compensatórios de cabeça durante a deglutição de sólidos.

A média do tempo para retomada da via oral exclusiva e retirada da sonda foi de quatro semanas, sendo que todos os pacientes iniciaram este retorno na consistência pastosa engrossada. Não foram observados sinais de aspiração em nenhum dos pacientes após a cirurgia. Por outro lado, 100\% dos pacientes apresentaram inapetência e perda de peso significativa após a cirurgia (mais de três quilos), mesmo com o uso de sonda nasoentérica e acompanhamento nutricional.

O teste não paramétrico de Kruskall Wallis (Teste $\mathrm{H}$ ) permite a comparação de dados com amostras diferentes. Este teste permitiu, portanto, a comparação dos achados pré- 
Tabela 1. Análise da fase oral da deglutição e do uso de via alternativa de deglutição antes e após a cirurgia

\begin{tabular}{|c|c|c|c|c|c|}
\hline Dados avaliados & & Pré-operatório & Pós-operatório & Total & Valor $\mathrm{p}$ \\
\hline \multirow{3}{*}{$\begin{array}{l}\text { Via alternativa de } \\
\text { alimentação }\end{array}$} & ausente & 9 & 0 & 9 & \multirow{3}{*}{$p=0,000$} \\
\hline & sonda & 0 & 5 & 5 & \\
\hline & Total & 9 & 5 & 14 & \\
\hline \multirow{4}{*}{ Mastigação } & não avaliado & 0 & 1 & 1 & \multirow{4}{*}{$p=0,037$} \\
\hline & eficiente & 5 & 0 & 5 & \\
\hline & ineficiente & 4 & 4 & 8 & \\
\hline & Total & 9 & 5 & 14 & \\
\hline \multirow{3}{*}{$\begin{array}{l}\text { Manipulação do bolo } \\
\text { alimentar }\end{array}$} & sem dificuldade & 8 & 2 & 10 & \multirow{3}{*}{$p=0,011$} \\
\hline & com dificuldade & 1 & 3 & 4 & \\
\hline & Total & 9 & 5 & 14 & \\
\hline \multirow{3}{*}{ Estase oral } & ausente & 9 & 3 & 12 & \multirow{3}{*}{$p=0,048$} \\
\hline & presente & 0 & 2 & 2 & \\
\hline & Total & 9 & 5 & 14 & \\
\hline \multirow{3}{*}{$\begin{array}{l}\text { Movimento } \\
\text { compensatório }\end{array}$} & não & 7 & 1 & 8 & \multirow{3}{*}{$p=0,044$} \\
\hline & $\operatorname{sim}$ & 2 & 4 & 6 & \\
\hline & Total & 9 & 5 & 14 & \\
\hline
\end{tabular}

Kruskall-Wallis (Teste $\mathrm{H}$ )

operatórios de nove pacientes, com os achados pós-operatórios de cinco pacientes.

Comparando-se os achados do pré-operatório com os achados do pós-operatório (Tabela 1) encontramos, de forma significativa $(\mathrm{p}<0,05)$, mudança da via de alimentação, que passou de uma alimentação exclusivamente oral, para uma alimentação exclusivamente enteral. Também houve mudança significativa na eficiência mastigatória, que passou a ser ineficiente em todos os pacientes. Durante a pesquisa da eficiência mastigatória no pós-operatório, cabe esclarecer que um paciente recusou-se a ingerir alimento sólido, motivo pelo qual encontramos uma linha na tabela com o achado "não avaliado".

Percebeu-se, de forma significativa a dificuldade dos pacientes em manipular o bolo alimentar durante o processo de mastigação, gerando dificuldade na formação de um bolo coeso. Isso demonstra que a cirurgia influencia na realização desta função, ou seja, a perda de parte da língua compromete o processo de mastigação.

A deglutição também foi prejudicada pela cirurgia, uma vez que o teste demonstrou de forma significativa a presença de estase oral após a deglutição e movimentos compensatórios de cabeça para a deglutição de alimentos sólidos.

\section{DISCUSSÃO}

O câncer de boca corresponde de 8 a 12\% dos casos de câncer diagnosticados em homens e mulheres, respectivamente ${ }^{(12,21)}$. A maior parte desses tumores origina-se em lábios, língua ou soalho bucal, sendo o tipo histológico mais comum o carcinoma espinocelular ${ }^{(12,21)}$, que acomete principalmente pacientes do sexo masculino, entre a $5^{\mathrm{a}}$ e $8^{\mathrm{a}}$ décadas de vida, sendo raro em indivíduos com menos de 40 anos de idade ${ }^{(22-23)}$. Nas mulheres, ocorre com maior freqüência na $6^{a}$ década de vida, na raça branca ${ }^{(24)}$. Os resultados encontrados nesta pesquisa confirmam os achados da literatura em relação ao tipo histológico mais comum e ao sexo e idade dos pacientes, uma vez que todos os pacientes eram portadores de carcinoma espinocelular e $67 \%$ da amostra eram homens com mais de 40 anos.

Os fatores de risco mais significativos encontrados na literatura para o surgimento do câncer de boca foram: o consumo de tabaco e de bebidas alcoólicas ${ }^{(8)}$. Os resultados presente estudo concordam com as referências da literatura, visto que $89 \%$ da amostra faziam uso tanto de tabaco quanto de álcool.

Sabe-se que a presença do câncer na cavidade oral pode comprometer os processos de mastigação e deglutição ${ }^{(25-26)}$. Estas funções podem se tornar mais alteradas após a cirurgia em função da retirada de estruturas, como a língua, fundamentais ao desempenho destas funções, gerando interferências no processo de alimentação. Dependendo das estruturas envolvidas na ressecção do tumor, as seqüelas na mastigação e/ou deglutição podem ser temporárias ou permanentes, sendo estas os principais impactos após a cirurgia. Estes impactos podem manifestar-se por meio de estase em cavidade oral, dificuldade no trânsito oral e aspirações antes, durante e após a deglutição $0^{(25-26)}$.

A mudança na via de alimentação do período pré-operatório para o período pós-operatório, ou seja, $100 \%$ dos pacientes passaram a alimentar-se por sonda nasoentérica $(\mathrm{p}=0,00)$, pode ser explicado pela necessidade de se aguardar o tempo de cicatrização das estruturas remanescentes sem a re-introdução de alimento, evitando, assim, o aparecimento de fístulas e infecção pós-operatória ${ }^{(27)}$.

A mudança na eficiência mastigatória $(\mathrm{p}=0,002)$ dos casos analisados pode ser explicada pela diminuição da eficiência dos golpes mastigatórios e pela dificuldade de manipulação do bolo alimentar dentro da cavidade oral $(\mathrm{p}=0,011)$ por falta de estrutura apresentada pelos informantes da pesquisa. $\mathrm{O}$ 
alimento, durante o processo de mastigação, deve ficar na face oclusal dos dentes, em função de um equilíbrio de forças entre a língua e o bucinador ${ }^{(2)}$. Havendo um desequilíbrio destas forças pela ausência da língua que faz a lateralização do bolo, a função de mastigação ficará prejudicada, justificando os achados deste estudo.

A deglutição também foi modificada pela cirurgia, pois houve estase em cavidade oral após a deglutição $(\mathrm{p}=0,048)$. Esta estase ocorreu provavelmente pela ausência de parte da língua, pois esta estrutura ajuda na limpeza do vestíbulo oral. A mudança nos movimentos de cabeça apresentados durante a deglutição de sólido $(\mathrm{p}=0,044)$ ocorreu, provavelmente, pela perda de parte da língua, fundamental para a ejeção do bolo alimentar.

Explica-se a integridade da musculatura mastigatória pelo fato destas estruturas não fazerem parte do campo operatório $^{(28)}$.

As alterações estatisticamente significantes confirmam os achados da literatura ${ }^{(18-19)}$ sobre as alterações de deglutição em glossectomizados parciais.

Apesar da reduzida amostra de pacientes, os resultados encontrados foram compatíveis com os dados da literatura. A dificuldade na captação destes pacientes pode ser atribuída a diversos fatores, como o não consentimento em participar da pesquisa, desistência após a cirurgia, mudança no procedimento cirúrgico com aumento do tamanho da ressecção e diagnóstico tardio, impossibilitando a realização da cirurgia parcial como modalidade de tratamento. Vale ressaltar que o tamanho da amostra reduzido não permite generalizações dos resultados, apesar da congruência com a literatura.

\section{CONCLUSÃO}

Os pacientes submetidos à glossectomia parcial apresentam, no pós-operatório recente, alterações significantes na mastigação e deglutição. Identificamos como mudança na função mastigatória o desenvolvimento de uma mastigação ineficiente e uma dificuldade de manipulação do bolo alimentar dentro da cavidade oral. Já com relação à deglutição, identificamos estase intra-oral e presença de movimentos compensatórios de cabeça.

Os resultados encontrados confirmam os achados da literatura, tanto com relação aos fatores etiológicos do câncer, quanto às possíveis alterações de mastigação e deglutição decorrentes da cirurgia curativa do câncer de língua.

\begin{abstract}
Purpose: To identify the commonest changes in chewing and swallowing as a result from curative tongue cancer surgery, with less than $50 \%$ of tongue resection and preservation of both mouth floor and tongue base. Methods: Nine patients - six men and three women - were assessed during the pre-surgical period, using a specific protocol. During the mediate postsurgical period, five patients - four men and one woman - were reassessed using the same protocol. The Kruskall Wallis non-parametric test (Test H) was used to analyze the significance of the results. Results: The comparison between pre and postoperative results showed significant $(\mathrm{p}<0,05)$ changes from oral to enteral feeding. There was also a significant change in chewing efficiency, which became ineffective in all patients. It was significantly observed the patients' difficulty in handling the food bolus during the chewing process, causing difficulty in forming a cohesive bolus. This demonstrates that surgery influences this function, that is, the partial loss of the tongue undermines the chewing process. The swallowing process was also impaired by the surgery: the protocol demonstrated significant presence of oral stasis after swallowing, and compensatory head movements for swallowing solids. Conclusion: The patients submitted to partial glossectomy presented significant alterations in chewing and swallowing as a result from surgical cancer treatment.
\end{abstract}

Keywords: Tongue neoplasms/complications; Mouth neoplasms/complications; Glossectomy; Stomatognathic system; Deglutition disorders/etiology; Mastication; Speech therapy

\title{
REFERÊNCIAS
}

1. Tanigute CC. Desenvolvimento das funções estomatognáticas. In: Marchesan IQ. Fundamentos em fonoaudiologia: aspectos clínicos da motricidade oral. Rio de Janeiro: Guanabara Koogan; 1998. p.1-6.

2. Bianchini EMG. Mastigação e ATM: avaliação e terapia. In: Marchesan IQ. Fundamentos em fonoaudiologia: aspectos clínicos da motricidade oral. Rio de Janeiro: Guanabara Koogan; 1998. p. 37-49.

3. Leopold NA, Kagel MC. Swallowing, ingestion and dysphagia: a reappraisal. Arch Phys Med Rehabil. 1983;64(8):371-3.

4. Macedo Filho ED. Conceitos e fisiologia aplicada da deglutição. In: Filho EM, Pisam JC, Carneiro J, Gomes G. Disfagia: abordagem multidisciplinar. 3a ed. São Paulo: Frôntis; 2000. p. 3-8.

5. Nicoletti G, Soutar DS, Jackson MS, Wrench AA, Robertson G. Chewing and swallowing after surgical treatment for oral cancer: functional evaluation in 196 selected cases. Plast Reconstr Surg. 2004;114(2):329-38.

6. INCA: Instituto Nacional do Câncer [Internet]. Rio de Janeiro:INCA; c2003. [citado 2005 Maio 6]. Disponível em: www.inca.gov.br/ estimativa/2005

7. Leite IC, Koifman S. Revisão dos fatores de risco para o câncer de boca e faringe. Rev Bras Cancerol. 1998;44(4):317-25.

8. Barbosa JF, Fonseca EP. Câncer bucal: diagnóstico, tratamento e reabilitação. Brasília: Ministério da Saúde; 1976.

9. Lopes MA, Coletta RD, Alves FA, Abbade N, Rossi Junior A. Reconhecendo e controlando os efeitos colaterais da radioterapia. Rev Assoc Paul Cir Dent. 1998;52(3):241-4.

10. Costa EG, Migliorati CA. Câncer bucal: avaliação do tempo decorrente 
entre a detecção da lesão e o início do tratamento. Rev Bras Cancerol. 2001;47(3):283-9.

11. Aksu G, Karadeniz A, Saynak M, Fayda M, Kadehci Z, Kocaelli H. Treatment results and prognostic factors in oral tongue cancer: analysis of 80 patients. Int J Oral Maxillofac Surg. 2006;35(6):506-13.

12. Grimaldi N, Sarmento V, Provedel L, Almeida D, Cunha S. Conduta do cirurgião-dentista na prevenção e tratamento da osteorradionecrose: revisão de literatura. Rev Bras Cancerol. 2005;51(4):319-24.

13. Elias R, Oliva Filho A, Barino B, Andrade T. Câncer bucal. JBC J Bras Clin Odontol Integr. 2002;6(31):25-8.

14. Neville BW, Day TA. Oral cancer and precancerous lesions. CA Cancer J Clin. 2002;52(4):195-215.

15. Ragghianti MS, Greghi SL, Capelozza AL, Resende DR. Tratamento radioterápico: parte I - reações adversas na cavidade bucal. Salusvita. 2002;21(1):77-86.

16. Signoretti GM, Trawitzki LVV. Seqüelas da radioterapia e metas da atuação fonoaudiológica no câncer de boca. J Bras Fonoaudiol. 2003;4(17):277-82.

17. Nemr K. Câncer de cabeça e pescoço. In: Marchesan, IQ. Fundamentos em fonoaudiologia: aspectos clínicos da motricidade oral. Rio de Janeiro: Guanabara Koogan; 1998. p. 85-90.

18. Furia CL, Carrara-de Angelis E, Martins NM, Barros AP, Carneiro B, Kowalski LP. Video fluoroscopic evaluation after glossectomy. Arch Otolaryngol Head Neck Surg. 2000;126(3):378-83.

19. Carrara-de Angelis E, Fúria CLB, Mourão LF. A fonoaudiologia no Hospital A.C. Camargo. In: Lagrotta MGM; César CPHAR. A fonoaudiologia nas instituições: terceira idade, hospital, escola, centro de saúde, clínica-escola, creche. São Paulo: Lovise; 1997. p. 170-4.

20. Pauloski BR, Rademaker AW, Logemann JA, Colangelo LA. Speech and swallowing in irradiated and nonirradiated postcirurgical oral cancer patients. Arch Otolaryngol Head Neck Surg. 1998;118(5):616-24.
21. Yuen APW, Wei WI, Won YM, Tang GK. Elective neck dissection versus observation in the treatment of early oral tongue carcinoma. Head Neck. 1997;19(7):583-8

22. Venturi BRM, Pampola ACF, Cardoso AS. Carcinoma de células escamosas da cavidade oral em pacientes jovens e sua crescente incidência: revisão de literatura. Rev Bras Otorrinolaringol. 2004;70(5):679-86.

23. Guebur MI, Rapoport A, Sassi LM, Oliveira BV, Pereira JCG, Ramos GHA. Alterações do fluxo salivar total não estimulado em pacientes portadores de carcinoma espinocelular de boca e orofaringe submetidos à radioterapia por hiperfracionamento. Rev Bras Cancerol. 2004;50 (2):103-8.

24. Amorim Filho FS, Andrade Sobrinho J, Rapoport A, Carvalho MB, Novo NF, Juliano Y. Estudo de variáveis demográficas, ocupacionais e co-carcinogênicas no carcinoma espinocelular de base de língua nas mulheres. Rev Bras Otorrinolaringol. 2003;69(4):472-8.

25. Magrin J, Kowalski LP, Carvalho AL. Carcinoma de boca. In: Carrarade Angelis E, Fúria CL, Mourão LF, Kowalski LP. A atuação da fonoaudiologia em câncer de cabeça e pescoço. São Paulo: Lovise; 2000. p. 61-6.

26. Logemann JA, Rademaker AW, Pauloski BR, Kahrilas PJ. Effects of postural change on aspiration in head and neck surgical patients. Otolaryngol Head Neck Surg. 1994;110(2):222-7.

27. Borges EL, Carvalho DV. Cuidados com fístulas e feridas. In: Salles JM, Freire AR, Vicente LC. Câncer de boca: uma visão multidisciplinar. Belo Horizonte: Coopmed; 2007. p.281-8.

28. Sousa AA, Chulam TC. Cirurgia do tumor primário inicial. In: Salles JM, Freire AR, Vicente LC. Câncer de boca: uma visão multidisciplinar. Belo Horizonte: Coopmed; 2007. p.77-84. 